\title{
Des modèles d'affaires circulaires pour valoriser des coproduits agricoles. Etude exploratoire de quatre initiatives
}

\section{Circular Business Models for Valorizing Agricultural By-products. Exploratory study of four initiatives}

\author{
Ilham-Soukaina Adim ${ }^{1}$, Mechthild Donner ${ }^{2}$, Fatiha Fort ${ }^{3}$, Romane Gohier ${ }^{4}$ \\ ${ }^{1}$ INRA, UMR MOISA, 2 Place Pierre Viala, 34060 Montpellier, France, soukaina.moulim@gmail.com \\ 2 INRA, UMR MOISA, 2 Place Pierre Viala, 34060 Montpellier, France, mechthild.donner@inra.fr \\ ${ }^{3}$ Montpellier SupAgro, UMR MOISA, 1101 Ave Agropolis, 34093 Montpellier, France, fatiha.fort@supagro.fr \\ ${ }^{4}$ INRA, UMR MOISA, 2 Place Pierre Viala, 34060 Montpellier, France, romane.gohier@inra.fr
}

\section{Remerciement}

Cet article a bénéficié du soutien de l'Union Européenne dans le cadre du programme de recherche et d'innovation Horizon 2020, projet NoAW ${ }^{1}$, grant agreement No 688338.

RÉSUMÉ. L'économie circulaire consiste à penser les systèmes de production en tenant compte de la fin de vie des produits. Elle constitue un nouveau modèle de pensée qui accompagne la transition vers le développement durable. Dans la gestion des déchets de l'agriculture, de nombreuses initiatives ont ainsi été mises en œuvre pour gérer les déchets et les coproduits afin d'optimiser la consommation des ressources et de réduire les impacts environnementaux de l'activité économique. Cet article propose une analyse du modèle économique de quatre initiatives de valorisation des coproduits de l'agriculture. Les résultats montrent que lorsque la valeur proposée correspond à un marché tangible l'approche du business model est basée sur la structure du marché et propose une valeur économique mais aussi sociale et environnementale. En revanche les initiatives où la valeur n'est pas marchande nécessitent un soutien de fonds publics et une participation des populations cibles. Cette recherche met également en évidence l'impact des caractéristiques des coproduits agricoles sur les business models circulaires et le caractère trans-sectoriel des activités de valorisation.

ABSTRACT. The circular economy consists of production systems that take into account the end of life of products. It is a new way of thinking that supports the transition towards sustainable development. Within the agricultural waste management domain, multiple initiatives have been started that manage waste and byproducts in order to optimise the consumption of natural resources and reduce the environmental impact of economic activities. This article discusses the business models and value propositions of four different initiatives, the aim of which is to valorize agricultural by-products. Results show that if the value proposed targets a direct market, the corresponding business model is based on the market structure and delivers economic as well as social and environmental value. In contrast, for initiatives offering value that is not guided by direct market logic, public financial support and the participation of target stakeholders are crucial. MOTS-CLÉS. Economie circulaire, modèles d'affaires, agro-déchets et coproduits, valorisation, développement durable, valeur.

KEYWORDS. Circular economy, business models, agro-waste and by-products, valorization, sustainable development, value.

\footnotetext{
${ }^{1}$ Guidé par une logique d'économie zéro déchet, le programme de recherche NoAW vise à développer des approches innovantes pour transformer les coproduits et déchets agricoles en ressources économiques et écologiques dans une logique d'économie circulaire (www.noaw2020.eu)
} 


\section{Introduction}

Face à l'exploitation massive et non raisonnée des ressources naturelles et à l'accroissement des préoccupations environnementales, le modèle économique linéaire de production « extraire, produire, consommer et jeter » est remis en question depuis quelques années. La transition vers l'Economie Circulaire (EC) apparaît dès lors comme une nécessité permettant d'optimiser la consommation des ressources et de réduire les impacts environnementaux de l'activité économique. L'approche par l'Economie Circulaire consiste à penser les systèmes de production différemment et à se préoccuper davantage du bouclage des flux de matière et d'énergie. Elle vise une gestion durable des ressources et une réduction de l'impact environnemental des activités de production. En effet, il s'agit d'écoconcevoir les produits et services, d'améliorer le réemploi, la réutilisation, l'allongement des durées d'usage, le recyclage et la restauration des ressources renouvelables [YIN 12].

Bien que ce concept ait fait l'objet d'une littérature particulièrement riche et en pleine expansion, il n'existe pas de définition normalisée à ce jour. L'EC se situe au croisement de plusieurs théories développées au sein de disciplines diverses depuis les années 1970 : écologie industrielle, cradle to cradle, économie de la performance, biomimétisme, économie bleue et conception régénérative [GAL 16]. La fondation MacArthur souligne que « l'EC est par nature réparatrice et régénérative et tend à préserver la valeur et la qualité intrinsèque des produits, des composants et des matériaux à chaque étape de leur utilisation. [...] La fondation considère que les flux de matières sont de deux types, des matières biologiques, qui ont vocation à retourner à la biosphère, et des matières techniques, qui ont vocation à circuler avec une perte de qualité aussi faible que possible. » [ELL 12, p. 2].

Dans cette perspective, le modèle circulaire consiste à penser les systèmes de production selon des boucles circulaires fermées de flux de matière, en marquant une rupture radicale avec les principes de production du modèle conventionnel. La transition vers ce nouveau modèle circulaire s'accompagne d'une mutation sur le plan structurel et organisationnel des systèmes de valeur et des flux de matière et d'énergie. Ces mutations font appel à de nouveaux Business Models (BM) afin de mieux intégrer les actions d'EC dans les stratégies d'entreprises.

Les systèmes agricoles et agroalimentaires doivent répondre aux besoins alimentaires, en quantité et qualité, d'une population croissante, mais également aux autres usages de la biomasse. Ils font pour cela face à des défis importants : acidification des sols, retour au sol insuffisant de la matière organique, stress hydrique et gaspillage alimentaire. Dans cet article, nous nous interrogeons sur la façon dont le secteur agricole peut participer à la transition vers l'EC grâce à la valorisation de coproduits et de déchets. Ces activités de valorisation reposent sur le principe d'EC et nécessitent la mise en place de BM spécifiques.

L'objectif de cette recherche est d'identifier les caractéristiques de ces BM et les conditions de succès qui permettent aux Business Models Circulaires de se développer dans le secteur de l'agriculture et de l'agroalimentaire. Nous analysons pour cela quatre initiatives de valorisation de coproduits de différentes filières agricoles dans la région Occitanie. 
L'Occitanie est la seconde région agricole française derrière la Nouvelle Aquitaine. Le secteur agricole et agroalimentaire joue un rôle majeur dans l'économie régionale : $2^{\mathrm{e}}$ secteur exportateur, il contribue largement au chiffre d'affaires régional et représente environ 160000 emplois. Les trois filières agricoles occitanes les plus importantes sont la vigne avec plus de 260000 ha, ce qui en fait le plus grand bassin viticole français; les céréales avec près de 800000 ha dédiés et l'élevage avec plus de 28000 exploitations [CHA 18]. Les initiatives sont sélectionnées parmi ces trois filières.

L'article est structuré comme suit. Dans un premier temps, nous introduirons le cadre théorique pour traiter d'une part l'EC dans le secteur agricole et agroalimentaire et d'autre part les spécificités de la construction des Business Models circulaires (BMC). La méthodologie de la recherche sera ensuite détaillée et les résultats de nos quatre cas analysés. Nous discuterons enfin les apports théoriques et les implications managériales et politiques de notre contribution.

\section{Cadre théorique}

\subsection{L'EC comme moyen de développement d'un système agricole circulaire}

Les systèmes agricoles performants nécessitent un entretien de la fertilité des sols; jusqu'au début du $\mathrm{XX}^{\mathrm{e}}$ siècle, les déjections animales et les résidus organiques des activités humaines constituent la principale source d'amendement. Les fumiers et résidus organiques des milieux ruraux sont réutilisés localement dans les fermes afin de produire des aliments, en partie exportés vers les villes. Celles-ci génèrent des résidus organiques comme les « boues », les « vidanges » et les sous-produits organiques issus d'activités artisanales ou industrielles [BAR 05] qui sont alors renvoyés vers les campagnes pour être réutilisés. Ces ressources sont considérées comme indispensables au développement agricole. Barles emploie le terme de "métabolisme » pour décrire cette relation entre ville et agriculture [BAR 05]. Au début du XX $X^{\mathrm{e}}$ siècle, l'augmentation des coûts de collecte et de traitement, la diversification des matières dans les déchets ménagers et les mouvements hygiénistes conduisent à considérer ces résidus organiques comme des déchets à éliminer. On assiste à une volonté politique de privilégier l'élimination à la réutilisation agricole. C'est le développement des engrais minéraux de synthèse qui permet de dégager l'agriculture de la nécessité de la fertilisation organique et d'établir une rupture avec les déchets urbains [JON 13]. La période de valorisation agricole massive des déchets urbains gagne un « sursis » avec la seconde guerre mondiale qui contraint à prolonger les anciennes méthodes. Mais, dès les années 1950, les engrais minéraux se généralisent et deviennent les principaux intrants fertilisants [JON 13].

Ainsi, l'agriculture française cherche une plus grande productivité et connaît de profondes mutations, tant techniques (mécanisation et motorisation, progrès agronomiques et scientifiques), que politiques (à l'échelle nationale puis européenne avec la Politique Agricole Commune) et économiques (généralisation de l'agriculture commerciale et mise en place des filières agroalimentaires) [RIE 09]. Ces mutations entraînent une concentration des exploitations et une spécialisation des systèmes de production. La polyculture, les activités de transformation et les techniques de recyclage des déchets sont progressivement 
abandonnées au profit d'une industrialisation de l'agriculture et d'une spécialisation dans la culture des produits les plus rémunérateurs nécessitant un apport d'intrants chimiques croissant. Selon Arnsperger et Bourg, Karl Marx mettait en lumière, dès le milieu du XIX siècle, «le caractère sur-exploiteur du capitalisme en arguant du caractère non circulaire de l'agriculture industrielle : les nutriments se voient extraits des sols sous forme de fibres alimentaires qui sont exportées vers les centres urbains et n'y retournent jamais. » [ARN 16, pp. 103-104]. Cette linéarité des processus de transformation d'intrants en extrants et des flux de consommation induit une non-soutenabilité de ce système agricole.

On constate un intérêt croissant pour une «circularisation» du secteur agricole et agroalimentaire. Considérés comme un levier d'optimisation du fonctionnement des acteurs au niveau d'un territoire [BEN 16], les systèmes agricoles sont capables de créer de nouvelles dynamiques à travers l'émergence de nouvelles opportunités et technologies, l'interaction locale entre les acteurs et la circulation des flux entre les diverses activités (élevage, production végétale, conditionnement des produits...). Appliquée au champ de l'agriculture et de l'alimentation, l'EC vise différents objectifs : la réduction des déchets, le bouclage des cycles nutritifs et l'évaluation du coût réel de la consommation des ressources naturelles [JUR 16]. Dans ce secteur, l'EC peut être visualisée selon trois niveaux; production agricole, consommation alimentaire et gestion des déchets et excédents [JUR 16]. Rood et al. [ROO 17] soulignent trois facteurs permettant aux secteurs agricoles et agroalimentaires d'atteindre un développement circulaire :

(1) Atteindre un usage et un management des ressources naturelles efficients et optimisés.

(2) Atteindre un usage optimal de l'alimentation en réduisant les pertes alimentaires, en consommant des produits moins transformés et en adoptant une consommation de protéines animales raisonnée.

(3) Atteindre un usage optimal des flux de résidus, en perdant aussi peu de biomasse que possible.

Nous nous intéressons dans cette recherche à ce dernier facteur et notamment à la transformation des agro-déchets en ressources. Pour atteindre un usage optimal des résidus et la circulation de la matière, il convient de développer de nouvelles technologies et des activités économiques ainsi que des outils de valorisation et de traitement des agro-déchets [SCH 13]. Ces activités de traitement et de valorisation constituent une préoccupation majeure du système agroalimentaire dans son ensemble, de par le volume considérable de déchets produits et le besoin d'investir dans des voies efficientes pour répondre aux besoins des futures générations d'une population croissante.En outre, les ressources contenues dans les agro-déchets représentent une grande quantité de biomasse valorisable à condition que leurs structures moléculaires soient abordées par une conversion appropriée en produits compétitifs. Les initiatives qui proposent de nouvelles voies de valorisation ou intègrent les déchets et coproduits dans les chaînes de production pour permettre leur réutilisation doivent être encouragées pour permettre la transition vers un système agroalimentaire circulaire [ULV 14]. La mise en place de telles activités de valorisation nécessite des 
innovations technologiques et organisationnelles, un changement institutionnel et une action entrepreneuriale [GHI 16].

Selon Callois [CAL 18], l'attrait des professionnels des secteurs agricoles et agroalimentaires ainsi que celui des politiques pour les thématiques d'EC est motivé par une volonté de relocaliser les emplois dans les zones rurales, garantir la continuité des activités et valoriser le potentiel de ressources non utilisées. Cependant, cet auteur considère que mettre en œuvre l'EC dans un environnement rural est complexe de par la dispersion géographique des activités. En effet, les configurations rurales sont hétérogènes, variées et propres à chaque territoire. L'éloignement géographique des gisements de coproduits a un fort impact en termes logistiques; l'activité de collecte et de massification peut donc nuire à la rentabilité économique d'un projet.

Afin d'appréhender les spécificités des activités de valorisation des coproduits agricoles, il est nécessaire de comprendre quelles sont les contraintes imposées par les caractéristiques intrinsèques de ces produits [FAH 16] :

(1) la saisonnalité : les volumes de produits agricoles varient à des intervalles réguliers et spécifiques à chaque production.

(2) la périssabilité : les produits agricoles évoluent au fur et à mesure du temps, leur durée de vie est limitée.

(3) la variabilité : il existe un écart entre le produit réel et le produit standard en termes de taille, couleur, courbe, etc. La teneur en molécules d'intérêt varie selon les saisons et les variétés de produits.

(4) la typicité géographique : les produits agricoles ont des qualités uniques qui reflètent un contexte territorial particulier, cette spécificité est due à des facteurs pédoclimatiques (températures, humidité, vent, ensoleillement, qualité du sol et de l'eau...), à des facteurs de production (pratiques agricoles, tradition...) et aux variétés spécifiques à des régions.

(5) le risque de contamination : les produits agricoles peuvent contenir des pathogènes ou des substances toxiques telles que des métaux lourds. Il y a un besoin de sécuriser les approvisionnements en termes de qualité.

(6) l'incertitude des prix [FAH 16]: bien que ce ne soit pas une caractéristique intrinsèque, la variation des prix des produits agricoles est récurrente et doit être prise en compte.

Ces éléments nous amènent à une analyse des conditions de succès ou d'échec des projets de développement de nouveaux Business Models et des mécanismes de création de valeur pour assurer le développement et la pérennité de ces activités. La section suivante permet de mieux spécifier le concept de Business Model et d'expliquer le mécanisme de création de valeur dans le cadre de l'approche circulaire, ainsi que d'identifier des cadres d'analyse pertinents.

\subsection{Les Business Models Circulaires}

La notion de valeur, au cœur des stratégies d'entreprises, est difficile à appréhender en raison de son caractère polysémique. Le BM propose un cadre d'analyse pour comprendre 
comment s'agencent les «constituants » de l'entreprise pour générer cette valeur. Il fait l'objet d'une ample littérature dans la sphère publique, académique, et dans les communautés liées au monde des affaires et de l'entreprise. Selon Osterwalder et Pigneur [OST 10], un BM est un outil conceptuel pour comprendre comment les entreprises créent, délivrent et capturent la valeur. Il vise à expliquer comment l'entreprise définit sa stratégie au travers des produits et services qu'elle propose à son marché, des revenus générés, des coûts associés, de sa stratégie de différenciation et de la façon dont elle intègre sa chaîne de production dans un réseau de valeur [RAS 07]. Lecocq et al. [LEC 06, p. 99] définissent le $\mathrm{BM}$ comme «les choix qu'une entreprise effectue pour générer des revenus ». Toutefois, l'approche du BM ne peut pas se limiter à la seule question de la création de la valeur économique. Selon Wirtz [WIR 11] le BM restitue la combinaison de facteurs de production nécessaires à la mise en œuvre de la stratégie d'entreprise et les fonctions des acteurs impliqués.

La littérature sur les Business Models Circulaires constitue un champ émergent dans le domaine du management [LIN 17, BEU 18]. Mentink [MEN 14, p. 24] les définit comme « la logique en fonction de laquelle une entreprise s'organise pour créer, délivrer et capturer de la valeur à partir de boucles fermées de matières ». Le BMC s'inscrit dans un système de boucles de matières fermées ou restauratrices. Cependant un BMC ne boucle pas nécessairement les flux de matière au sein des frontières de son système mais prend part à un ensemble qui permet de boucler les flux [MEN 14; ANT 16]. Ainsi, Linder and Williander [LIN 17, p. 183] considèrent le BMC comme « un modèle d'entreprise dans lequel la logique conceptuelle de la création de valeur est basée sur l'utilisation de la valeur économique retenue dans les produits après utilisation dans la production de nouvelles offres ».

Micheaux et Aggeri [MIC 17] différencient le BMC du BM classique par son objectif principal. Contrairement aux BM classiques, l'objectif central du BMC n'est pas la performance économique, mais le bouclage des flux d'énergie et de matière. La bonne santé financière de la structure est seulement nécessaire pour assurer la pérennité de l'activité.

Blockpoel [BLO 16] identifie différentes sources de création de valeur dans les BMC. La première est l'avantage concurrentiel généré par la gestion de l'environnement et l'amélioration de ses capacités organisationnelles (vision partagée, prise en compte des parties prenantes et proactivité stratégique). La seconde source de création de valeur est une source de revenus supplémentaires grâce à la valorisation du potentiel des produits en fin de vie. Différentes stratégies sont alors possibles : les déchets peuvent être utilisés comme ressources ou peuvent être vendus en évitant ainsi les coûts d'élimination.

Dans l'optique de comprendre le fonctionnement des BMC, de caractériser leurs spécificités et de déterminer les logiques de création de valeur, un cadre d'analyse est nécessaire. On observe deux courants principaux : la caractérisation statique qui permet d'analyser le $\mathrm{BM}$ à un instant $\mathrm{t}$ et le processus dynamique qui permet de souligner les évolutions qui découlent des choix des entreprises. Pour qualifier les BM classiques, la littérature, comparant de nombreux cadres d'analyse des BM [BEU 18], [GAR 17], s'accorde sur la pertinence de deux outils principaux : le modèle de Demil et Lecocq [DEM 10] qui souligne les relations entre les éléments du BM et les mécanismes d'évolution, et le modèle Canvas d'Osterwalder et Pigneur [OST 10] qui décrit le BM à un instant t selon 
l'analyse de neuf éléments : la proposition de valeur, les activités clés, les partenaire clés, les ressources clés, les segments de clients, la relation clients, les canaux, les coûts et les sources de revenus.

Certains cas étudiés dans notre recherche sont très récents et leurs BM ont peu évolué depuis leur création, le cadre d'analyse dynamique ne semble donc pas pertinent dans leur contexte. L'objectif de ce travail est la caractérisation des BMC dans le secteur agroalimentaire. Le modèle statique propose une description plus complète et détaillée de tous les composants du BM, et cette approche a donc été retenue pour nos études de cas. Cependant, cet outil conceptuel généraliste ne permet pas de révéler toutes les spécificités des BMC.

Différents travaux académiques ont été récemment conduits pour adapter le modèle Canvas aux BMC en incluant des éléments spécifiques à l'EC. Trois cadres d'analyse des BMC proposés par différents auteurs nous apparaissent particulièrement pertinents : Mentink [MEN 14], Lewandowski [LEW 16] et Sempels [SEM 14]. The Business cycle Canvas model [MEN 14] propose d'adapter graphiquement à chaque BMC la logique de création de valeur basée sur la fermeture des boucles afin de souligner les stratégies de circularité. Il permet de comprendre comment la valeur est co-créée avec les partenaires et les parties prenantes. Le modèle de Lewandowski [LEW 16] complète le modèle Canvas classique par deux éléments: les facteurs d'adoption (facteur internes et externes influençant l'adoption des stratégies de circularité) et le système de reprise (stratégie de bouclage des flux). Le modèle de Sempels [SEM 14] ajoute quant à lui trois composantes au modèle Canvas: l'efficacité et l'efficience de l'organisation, les externalités positives et négatives ainsi que les vecteurs de productivité. La structure et les composants du modèle de Mentink [MEN 14] doivent être adaptés à chaque cas selon les stratégies de circularité choisies, ce modèle rend donc la comparaison de différents cas plus difficile. Les modèles de Lewandowski et de Sempels ne permettent pas de mettre en évidence les relations avec les parties prenantes et les résultats en termes de durabilité. Ainsi, l'identification de cadres d'analyse a été étendue aux Business Models durables. The triple layered business model [JOY 16] est composé des neuf éléments du modèle Canvas, analysés selon les trois dimensions du développement durable : économie, social et environnement. Le volet économique correspond au modèle Canvas original, le volet social permet une prise en compte de toutes les parties prenantes et le volet environnemental s'appuie sur l'approche de l'analyse du cycle de vie afin de tenir compte des impacts environnementaux à chaque étape de la vie du produit. L'EC s'axe principalement sur la recherche d'amélioration des impacts environnementaux et le volet social apparaît trop complet pour caractériser les externalités sociales des BMC. Antikainen et Valkokari [ANT 16] proposent un modèle appelé Sustainable Circular Business Model Innovation, adapté du modèle Canvas. Ce cadre d'analyse inclut des éléments pour prendre en compte l'environnement de l'entreprise et ses parties prenantes. Il intègre également une analyse des impacts environnementaux, sociaux et économiques. La littérature sur l'EC souligne l'importance du contexte et des réseaux d'acteurs dans la mise en place de stratégies de circularité. L'ajout de ces éléments est donc nécessaire pour comprendre le fonctionnement des BMC. L'analyse des impacts est basée sur les travaux de Joyce et Paquin [JOY 16] et l'approche Triple Bottom Line d'Elkington [ELK 98]. Cette approche consiste à évaluer le résultat d'une entreprise selon sa performance sociale, environnementale et économique (People, Planet \& Profit); ce qui 
permet ainsi de présenter de manière synthétique les externalités en termes de développement durable.

Nous supposons que, pour le secteur agricole/agroalimentaire, les politiques et lois sont particulièrement importantes et dynamiques et que les exigences des parties prenantes sont plus fortes que dans d'autres secteurs comme, par exemple, celles des consommateurs quant aux questions de durabilité et de santé. Bien que s'intéressant à l'ensemble des Business Models durables, le modèle d'Antikainen et Valkokari [ANT 16] nous paraît être le plus adapté à notre étude pour différentes raisons : comme vu précédemment, les BMC sont interconnectés à d'autres acteurs et ce modèle permet de souligner la prise en compte et l'implication de ces acteurs. De plus, il requiert une analyse des évolutions de l'environnement de l'entreprise qui peuvent représenter des facteurs d'adoption externes [LEW 16] ou, au contraire, représenter un frein au développement de l'activité. Cet aspect est indispensable à étudier dans le secteur agricole/agroalimentaire au vu du dynamisme des obligations des entreprises et de l'évolution des mentalités des consommateurs [JUR 16]. Enfin, les impacts en termes de durabilité demandent une comparaison des obligations et des bénéfices retirés du procédé de valorisation. Cela permet ainsi de comprendre l'intérêt de la proposition de valeur en complément de l'évaluation économique (coûts et revenus) dans une logique de triple bottom line.

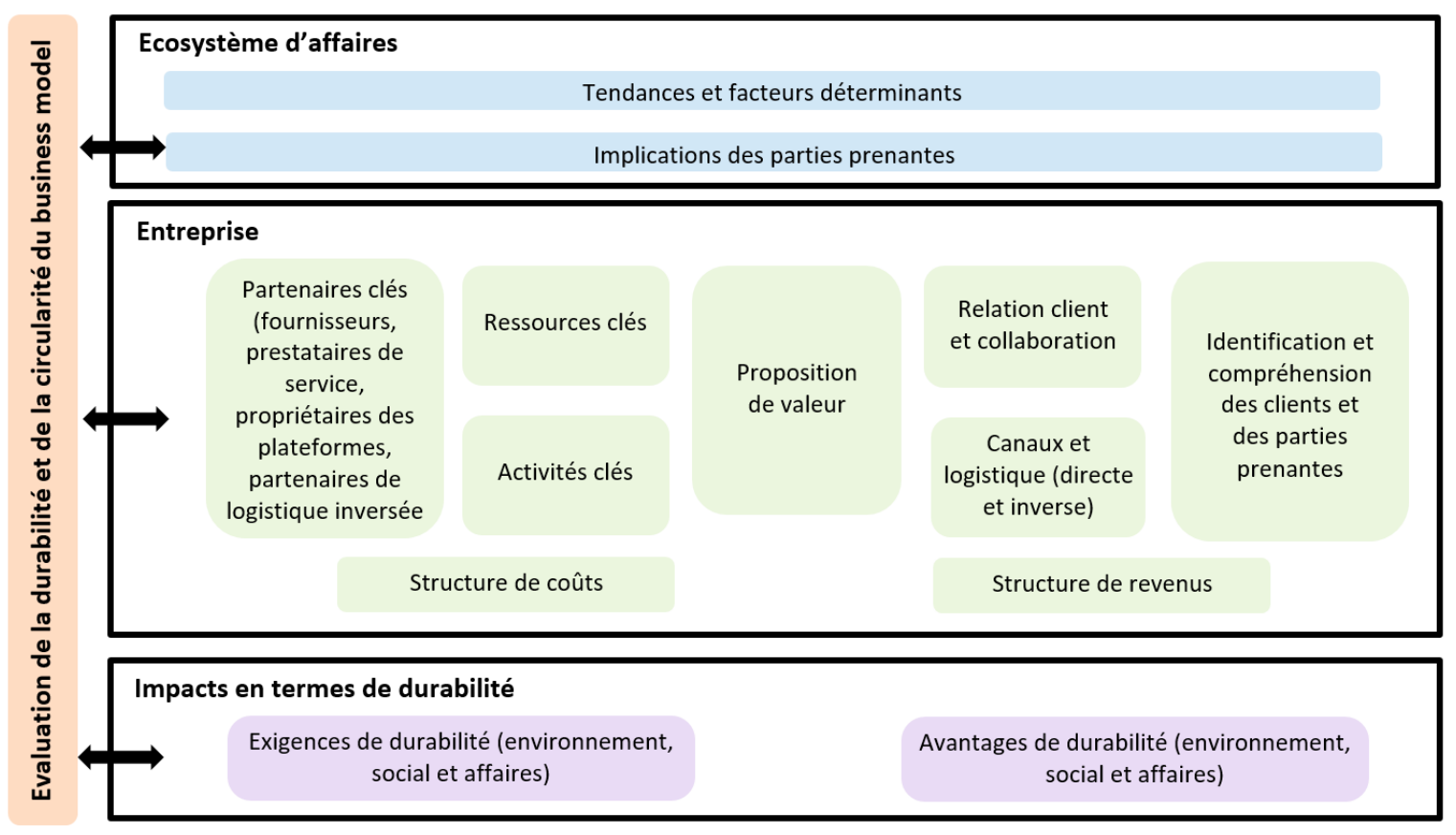

Figure 1. Circular Business Model Innovation (Antikainen et Valkokari, 2016), traduit de l'anglais par l'auteur

Nous choisissons donc le cadre d'analyse Circular Business Model Innovation (figure 1), proposé par Antikainen et Valkokari, pour structurer l'analyse des BMC de nos études de cas. Il nécessite l'étude de différents éléments :

(1) Evolution de l'environnement: politique, économique, social, technologique, écologique et législatif 
(2) Prise en compte des parties prenantes : parties prenantes liées à l'activité et influence des réseaux d'acteurs

(3) Partenaires clés : fournisseurs, pérennité et qualité des relations, variété des partenaires, mutualisation d'infrastructures ou d'expertises, co-création

(4) Activités clés : activités logistiques, de transformation, de valorisation, équilibre externalisation/internalisation

(5) Ressources clés : humaines, intellectuelles, technologiques

(6) Proposition de valeur: offre de produits et services, problèmes que permet de résoudre la proposition de valeur, éléments différenciant par rapport à la concurrence, degré d'innovation

(7) Relation client : marque, solidité des relations, information du consommateur

(8) Canaux : canaux de distribution, canaux de communication et logistique inversée

(9) Segments de clients : clients professionnels et/ou particuliers, type de segmentation des clients

(10) Coûts : prévisibilité des coûts, économies d'échelles, investissements

(11) Sources de revenus : stratégie de prix, trésorerie, diversification des revenus, aides financières

(12) Exigences de durabilité : réglementations, lois, obligations envers les parties prenantes

(13) Impacts en termes de durabilité : impacts économiques, environnementaux et sociaux

Il existe peu d'évaluations sur les impacts de durabilité de l'EC. Les effets environnementaux commencent à être pris en compte mais leur mesure reste encore difficile, la plupart des études privilégiant un indicateur au lieu d'utiliser une batterie d'indicateurs. Les effets sur le développement social territorial ne sont pas mesurés actuellement [GAL 16].

\section{Méthodologie}

Les études de cas présentées ici sont exploratoires compte tenu du peu de travaux académiques dans le domaine [ANT 16, BEU 18]. L'étude de cas est reconnue comme une méthode permettant de comprendre des phénomènes contemporains et peu investis par les chercheurs ; surtout «lorsque les frontières entre le phénomène et le contexte ne sont pas clairement évidentes, et pour laquelle de multiples sources de données sont utilisées » [YIN 03, p. 25].

En effet, notre approche multi-cas implique une étude d'un nombre limité d'unités afin de comprendre une plus grande classe d'unités (similaires), observées à un moment donné ou pendant une période délimitée. Elle est essentiellement descriptive, consiste en une démarche exploratoire et se focalise sur la complexité du cas concret sans prétendre le généraliser [RID 12].

L'étude a été réalisée dans le cadre du programme de recherche NoAW qui s'intéresse aux innovations technologiques de valorisation des déchets de 3 filières agricoles : les céréales, l'élevage (fumier) et le vin. L'ancienne région Languedoc-Roussillon, aujourd'hui intégrée à la région Occitanie, a été sélectionnée comme l'un des terrains d'études du projet. Les études de cas ont donc été sélectionnées dans cette région et à proximité. Le but est à la 
fois de diversifier le secteur d'activité des initiatives et de pouvoir établir une vision plus large sur les actions circulaires entreprises en agriculture :

- Une valorisation des déchets sous forme de biogaz dans la coopérative «Les bergers du Larzac »

- Une industrie de valorisation des résidus de vinification «Union de coopératives Grap'Sud»

- Une utilisation de la paille dans une association «Bâtir en Balles »

- Un agro pôle de services divers de valorisation des déchets par une association

«Bio Vallée Lauragais ».

Pour recueillir les données, nous nous sommes d'abord appuyés sur les sites internet des initiatives et des articles de presse, puis nous avons utilisé un guide d'entretien qualitatif développé dans le cadre du projet NoAW, servant de base pour orienter les entretiens menés avec les dirigeants ou responsables de chacune des unités de valorisation des agro-déchets. Ces entretiens ont porté principalement sur l'historique et le développement des activités pour comprendre la vision des acteurs engagés dans cette démarche et les enjeux de cette intégration, les agro-ressources valorisées, la coopération entre acteurs au sein de leurs territoires, le financement et les facteurs ayant favorisé ou entravé le succès de l'initiative. Le questionnaire s'appuyait sur des questions ouvertes laissant l'opportunité aux interviewés de s'exprimer sur leur propre expérience. Ces entretiens, en face à face, ont duré entre une et deux heures et demie, et ont fait l'objet de comptes rendus individuels, puis ont été croisés dans le cadre d'une analyse thématique pour intégrer l'ensemble des informations recueillies.

\section{Résultats}

En premier lieu, nous présentons le BM de chaque cas étudié. Des caractéristiques communes et des dissemblances sont ensuite soulignées afin de pouvoir mettre en lumière deux catégories de BMC. En second lieu, une caractérisation des BMC sera proposée selon le cadre d'analyse Sustainable Circular Business Model Innovation, développé par Antikainen et Valkokari [ANT 16].

Grap'Sud est une union de coopératives créée en 2006 et issue de plusieurs distilleries coopératives plus anciennes, initiées pour répondre aux exigences légales de traitement des déchets de la vinification. Son activité principale est la valorisation de coproduits de la vinification et de la culture de fruits (raisin, olives). L'union propose un large portefeuille de produits innovants et biosourcés, qu'elle développe dans une logique de valorisation en cascade. Elle propose également des services connexes tels que la désalcoolisation, la filtration et l'extraction de molécules à forte valeur ajoutée. Ses revenus sont basés sur la vente de produits et services précédemment cités, auxquels s'ajoutent des aides publiques. L'union adopte des stratégies de commercialisation différenciées selon les marchés ciblés, elle possède ainsi un portefeuille de BM. Elle adopte une démarche de co-création des produits avec certaines entreprises clientes, et établit également des partenariats avec des établissements de recherche et des entités publiques. Son développement a été rendu possible grâce à des ressources clés, tant humaines (210 employés), technologiques (six unités de production implantées dans les bassins viticoles, investissement en infrastructures 
et procédés innovants) qu'intellectuelles (brevets). Grap'Sud collecte les coproduits et déchets directement chez les producteurs. Bien que ces derniers les cèdent gratuitement, le coût d'acquisition est non négligeable en raison des frais logistiques induits :

«Parfois les coûts de production à partir des déchets sont plus importants que si on travaillait directement le raisin. Ce n'est pas parce que le déchet est gratuit que les coûts sont faibles. » (Directeur marketing).

Le second enjeu relatif à la récupération de déchets est l'évaluation de la quantité de matière disponible : l'entreprise Grap'Sud s'est implantée dans un premier temps au sein de zones viticoles françaises pour un bouclage de flux local mais collecte désormais des coproduits en Espagne pour bénéficier de quantités suffisantes pour la valorisation. Arrivés dans les unités de valorisation, les coproduits sont d'abord prétraités, les molécules d'intérêt sont extraites sur la matière fraîche, puis le reste de la matière est valorisée sous différentes formes (alcool brut, spiritueux, huiles essentielles, sirop de fruits, alimentation animale, engrais, etc.). Ses clients sont des professionnels et sont segmentés par secteur : industrie agroalimentaire, industrie neutraceutique, nutrition animale, agriculture et agro-industries, œnologie, alcools et spiritueux. L'union a créé différentes marques adaptées à chaque filière de vente. Grap'Sud a un impact environnemental positif important grâce à une valorisation efficiente de la biomasse qui permet d'éviter un volume conséquent de déchets et de proposer des produits biosourcés en substitution de produits habituellement issus de la pétrochimie (comme de la poudre colorante d'anthocyane remplaçant des colorants de synthèse). De plus, l'activité économique générée dans ses différentes distilleries améliore le dynamisme économique de ces territoires et créé des emplois locaux non délocalisables. Les axes de développement sont conduits dans une logique de marché. Selon le Directeur marketing, lorsque la $\mathrm{R} \& \mathrm{D}$ a permis de développer une nouvelle voie de valorisation, sa commercialisation dépend de son attractivité sur le marché :

«On regarde le marché actuel et potentiel en fonction des produits de substitution, des prix et de la création de valeur. » (Directeur marketing).

«Les bergers du Larzac » est une coopérative créée en 1996 et réunit 32 adhérents coopérateurs dont la production de fromage est mutualisée. Les adhérents participent à la gouvernance de la coopérative et définissent les axes stratégiques. Face à des contraintes réglementaires pour le traitement des rejets de la production et en raison de l'éloignement géographique des acheteurs potentiels de lactosérum, la coopérative a développé en 2010 une infrastructure de valorisation des déchets et effluents par méthanisation, produisant du biogaz, de la chaleur et un digestat. Le biogaz et la chaleur récupérés permettent de chauffer le digesteur de méthanisation, le lait, les bains-marie des cuves à fromages, les solutions de lavage et l'eau sanitaire :

«L'objectif est d'assurer une autonomie en énergie et une réduction de la facture d'électricité : le projet (capacité de $400 \mathrm{~m}^{3}$ ), en permettant le stockage, réduira la facture de gaz de 50\% » (Directeur des Bergers du Larzac).

Le digestat est fourni à un de ses partenaires, la Fumière, qui le transforme en compost que les producteurs réutilisent ensuite. Le second partenaire important de la coopérative est le prestataire du procédé de méthanisation, avec lequel le projet a été co-construit. La 
stratégie de circularité mise en place repose donc sur la méthanisation qui permet de boucler les flux d'énergie et de nutriments. Cette infrastructure a demandé un investissement financier important, réalisé en deux étapes successives, et a été soutenue par des aides publiques à hauteur d'environ $25 \%$, mais a également nécessité une montée en compétence du personnel sur ce procédé. Le projet a été développé comme solution locale de traitement des déchets et effluents de la fromagerie, mais permet plusieurs externalités positives. D'un point de vue environnemental, le bouclage des flux et le retour au sol des nutriments réduisent les déchets et les émissions polluantes. Un emploi a été créé pour la gestion de la méthanisation, l'impact social est donc également positif. D'un point de vue économique, la réduction de la facture énergétique est un avantage pour les adhérents et cela représente également un argument important pour attirer de nouveaux producteurs dans la coopérative. Face au succès de ce projet de méthanisation, une réflexion est en cours pour imaginer :

«Un système de gestion des déchets incluant toute la filière depuis l'amont jusqu'à l'aval (déchets des fermes, de transformation, emballage...) »(Directeur des Bergers du Larzac).

L'association Bâtir en balles a été fondée en 2015 par le créateur d'une association d'écomatériaux à base de coques de riz et un agriculteur détenant une entreprise spécialisée dans les panneaux d'isolation thermique. L'association a pour but de développer la valorisation des coproduits agricoles de céréales dans le secteur de la construction. Elle participe à l'élaboration d'agro-matériaux innovants : béton isolant, enduit terre, panneaux décoratifs et isolant en vrac. Bâtir en balles se positionne comme un facilitateur entre les détenteurs de coproduits céréaliers et le secteur de la construction. Elle intervient auprès des décortiqueurs pour adapter leurs procédés et faciliter la réutilisation des balles de céréales, mais également auprès des acteurs de la construction pour promouvoir les agro-matériaux développés. Elle collabore également avec des écoles d'ingénieurs. En sus de ses activités de coordination, Bâtir en balles réalise également des analyses de balles et des prestations de conseil technique, grâce à son équipe de bénévoles. Les frais de fonctionnement de l'association sont couverts, en partie seulement, par les cotisations de ses 12 membres. Cependant, le modèle financier actuel ne permet ni de financer des employés ni certaines actions coûteuses telles que la normalisation des agro-matériaux. L'absence d'aides publiques est liée à l'absence de reconnaissance de cette technique par rapport aux procédés de valorisation plus développés de la paille et du chanvre :

«Il faut développer d'abord la notoriété afin de pouvoir surmonter ce frein financier» (Secrétaire général).

L'association est à but non lucratif, elle se positionne comme une structure d'appui et d'accompagnement. Les impacts économiques, environnementaux et sociaux sont relativement faibles au sein de ses frontières mais elle permet d'initier de nouveaux concepts économiques basés sur la valorisation de coproduits de céréales. Le potentiel de cette activité est important et permettrait d'éviter une grande quantité de déchets, de favoriser l'éco-construction, de créer des activités économiques ainsi que de nouveaux emplois. Bâtir en balles est donc un maillon qui permet le développement de filières circulaires. 
Le projet Bio-Vallée Lauragais, initié en 2009, visait d'abord à former un cluster regroupant des producteurs et des industriels positionnés sur les thématiques de l'alimentation biologique et du développement durable. Par la suite, l'association BioVallée Lauragais (BVL) a été créée en 2011 avec l'objectif depuis 2016 de développer l'économie circulaire au sein de la vallée du Lauragais :

«Ce changement d'orientation a eu lieu car une demande de financement n'était plus justifiée par le bio uniquement » (Coordinatrice de BVL).

Cette association compte deux salariés, elle a pour mission de piloter les projets de l'agro-parc et de coordonner les différents acteurs de la zone. Dans une logique d'animation territoriale, l'association met en réseau un ensemble d'acteurs différents pour renforcer leurs synergies et structurer de nouvelles filières circulaires. Grâce à son expertise en écologie industrielle et territoriale, à sa légitimité et à son capital relationnel, elle peut se positionner comme une structure d'appui technique et organisationnel intégrative et innovatrice :

« Nous n'avons pas de modèle pour notre initiative. Nous sommes peut-être des pionniers » (Gérant de Cler-Verts, entreprise membre de BVL).

Bio-Vallée Lauragais organise des rencontres (comme les rencontres régionales de l'économie circulaire) et des formations, réalise de la veille technologique et commerciale et recherche des porteurs de projets à fort capital synergique avec les acteurs de la zone afin de renforcer la dynamique circulaire. La santé financière de l'association est fragile et repose principalement sur des aides financières publiques. L'association a d'ailleurs été mise en sommeil en janvier 2018. De même que Bâtir en balles, Bio-Vallée Lauragais aurait besoin d'une structure de financement solide pour pouvoir pérenniser ses activités. L'association a néanmoins permis de développer de nombreuses synergies de substitution et de mutualisation entre les acteurs, favorisant ainsi la valorisation de déchets et la réduction d'utilisation de ressources. Elle a également permis de dynamiser la zone d'un point de vue économique en attirant de nouveaux acteurs, créant ainsi de nouveaux emplois dans la vallée du Lauragais :

«Le projet défend un fort ancrage local et participe à la promotion du territoire, par l'approvisionnement local et l'intégration d'un grand nombre d'acteurs et de structures locales » (Coordinatrice de BVL).

La compréhension de ces quatre BM permet de mettre en lumière des caractéristiques communes : ces initiatives ont toutes en commun une préoccupation environnementale et adoptent les principes de l'EC. Ces BM conçus autour des filières de valorisation d'agrodéchets génèrent de fortes interdépendances avec des acteurs de la recherche, des collectivités publiques, des acteurs en amont et en aval de la filière. De plus, les entretiens réalisés soulignent la volonté de créer une valeur économique, sociale, environnementale et territoriale. Grâce à l'analyse des BM de ces projets, nous pouvons catégoriser les BMC en deux groupes distincts : les modèles marchands et les modèles non marchands.

Grap'Sud et Les bergers du Larzac ont des modèles économiques marchands : via des unités d'extraction et des processus de méthanisation, ces entreprises valorisent des coproduits pour les transformer en produits commercialisables. Elles génèrent ainsi une 
valeur ajoutée, créent des emplois et permettent de réduire les déchets de la production agricole. La circulation des flux, au sein de leurs frontières, permet de constituer des boucles fermées de matière et d'énergie ; leur impact environnemental est donc important. La vente des produits créés, ou leur utilisation, génère des revenus ou permet une réduction des coûts de production. Il apparaît néanmoins que des aides financières publiques ont été nécessaires pour concrétiser leurs actions. Ces deux entreprises collaborent avec différents partenaires (acteurs économiques en aval et en amont de leur filière, acteurs publics et centres de recherche) pour co-construire leurs projets de valorisation. Nous constatons ainsi de nombreuses caractéristiques communes à ces deux BMC que nous pouvons synthétiser en utilisant le cadre d'analyse choisi [ANT 16] dans le tableau 1.

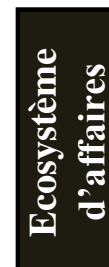

Évolution de l'environnement : contraintes réglementaires sur le traitement des déchets, incitations politiques, attrait pour les produits biosourcés, évolution technologique, compétition à l'innovation, variabilité des volumes de déchets

Prise en compte des parties prenantes : co-construction avec les acteurs de la filière amont et/ou aval, implication des partenaires, importance des adhérents dans la gouvernance pour le modèle coopératif

\begin{tabular}{|c|c|}
\hline $\begin{array}{c}\text { Partenaires } \\
\text { clés : } \\
\text { collectivités } \\
\text { territoriales } \\
\end{array}$ & centres de \\
& recherche \\
& acteurs de la \\
& filière amont \\
pour répondre à \\
leurs contraintes \\
de gestion des \\
coproduits \\
\hline 0
\end{tabular}

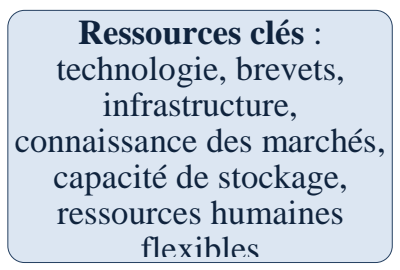

Activités clés :

transformation de coproduits en énergie ou en produits à valeur ajoutée, logistique, stockage

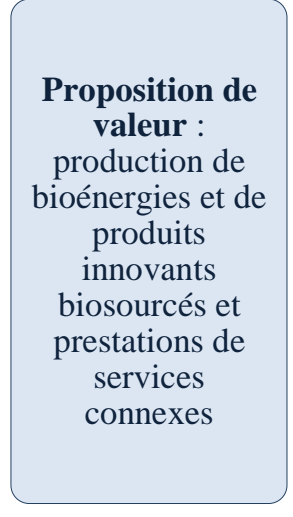

Relation client : cocréation et relation de long-terme

Canaux : logistique de récupération des coproduits
Segments de clients : utilisation interne ou vente à des tiers

Segmentation des clients par secteurs d'activité

Structure de coûts : investissement en R\&D et en technologie, frais de logistique inversée, coût de traitement, coût des ressources humaines, coût des brevets et licence de savoir-faire

Sources de revenus : vente de produits ou services, réduction du coût de disposition des déchets et de consommation énergétique, aides financières publiques

Exigences de durabilité : réponse à des contraintes réglementaires, solution de valorisation locale, volonté d'écoconception (produits et procédés non polluants), intérêt économique pour assurer la pérennité de l'activité

Impacts en termes de DD : réduction du volume de déchets générés, retour au sol des nutriments, valorisation en cascade ou cogénération, complémentarité d'usage, dynamisme économique local, création d'emplois non-délocalisables

\section{Tableau 1. Caractéristiques communes aux BMC marchands}

Bâtir en balles et Bio-vallée Lauragais sont des associations qui relèvent du modèle non marchand. Elles sont à but non lucratif, et se positionnent comme des structures d'appui et d'accompagnement. Un BMC ne boucle pas nécessairement les flux de matière au sein des frontières de son système mais prend part à un ensemble qui permet de boucler les flux. Ces deux associations s'inscrivent dans cette idée, bien que leurs impacts économiques, sociaux et environnementaux soient limités au sein de leurs frontières, elles sont nécessaires au développement de nouveaux concepts économiques et à la création de filières circulaires. 
Elles ont été créées à l'initiative d'acteurs hétérogènes dans le cadre de projets d'EC, et interviennent pour faire le lien entre des acteurs habituellement cloisonnés. La mise en place de synergie entre ces acteurs permet de créer une valeur nouvelle et multidimensionnelle qui répond à des enjeux économiques, sociétaux, environnementaux mais aussi territoriaux. En effet, elles participent au développement local et permettent de mettre en œuvre des projets d'EC inter-acteurs en nouant des partenariats avec de nombreux acteurs économiques. Elles ont pour point commun leur fragilité financière. Leurs activités de prestation de service restent mineures, ces associations sont dédiées à la coordination de projets entre de nombreux acteurs, qu'il convient de qualifier de populations cibles et non de clients. Il parait nécessaire d'innover pour amener ces populations cibles à s'engager en participant aux frais de fonctionnement de ces associations. Nous pouvons synthétiser les caractéristiques des BMC non marchands de nos cas empiriques dans le cadre d'analyse proposé par Antikainen et Valkokari [ANT 16] (tableau 2).

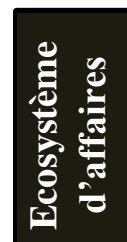

Évolution de l'environnement : développement de cluster ou de réseau d'affaires, acteurs cloisonnés mais à fort potentiel synergique, volonté politique de développer l'EC et les synergies d'écologie industrielle et territoriale

Prise en compte des parties prenantes : implication des parties prenantes dans le développement de projets et la prise de décision

\begin{tabular}{|c|c|c|c|c|c|c|}
\hline \multirow[t]{2}{*}{ 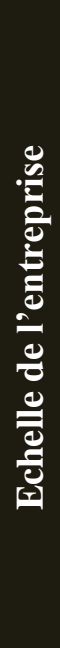 } & $\begin{array}{l}\text { Partenaires } \\
\text { clés : } \\
\text { partenariats } \\
\text { avec des entités } \\
\text { publiques, des } \\
\text { centres de } \\
\text { recherches et } \\
\text { des acteurs } \\
\text { multi-filières }\end{array}$ & \begin{tabular}{|} 
Ressources clés : \\
compétences \\
internes, capital \\
relationnel, réseau, \\
connaissance des \\
marchés et des \\
populations cibles \\
Activités clés : veille, \\
formation, animation, \\
appui technique, \\
développement de \\
partenariats
\end{tabular} & \multicolumn{2}{|c|}{$\begin{array}{l}\text { Proposition de } \\
\text { valeur : } \\
\text { développement de } \\
\text { stratégies de } \\
\text { circularité inter- } \\
\text { acteurs, } \\
\text { coordination de } \\
\text { projets, animation } \\
\text { de réseau }\end{array}$} & \begin{tabular}{|c|}
$\begin{array}{c}\text { Relation avec les } \\
\text { populations cibles : } \\
\text { co-construction des } \\
\text { démarches, } \\
\text { consultation }\end{array}$ \\
$\begin{array}{c}\text { Canaux : canaux } \\
\text { de communication } \\
\text { diversifiés, } \\
\text { relation en face à } \\
\text { face }\end{array}$
\end{tabular} & $\begin{array}{l}\text { Segments de } \\
\text { populations } \\
\text { cibles : } \\
\text { variables selon les } \\
\text { projets : par } \\
\text { filière, par } \\
\text { territoire, par type } \\
\text { d'acteurs } \\
\text { économiques, par } \\
\text { catégorie de } \\
\text { déchets ou } \\
\text { coproduits }\end{array}$ \\
\hline & $\begin{array}{r}\text { Structure de c } \\
\text { coor }\end{array}$ & $\begin{array}{l}\text { ûts : frais de fonctionne } \\
\text { ination et d'animation }\end{array}$ & & $\begin{array}{r}\text { Sour } \\
\text { struc } \\
\text { (cotisat }\end{array}$ & $\begin{array}{l}\text { es de revenus : fragilit } \\
\text { Ires, participation des p } \\
\text { ns, dons), vente de pre } \\
\text { subventions financ }\end{array}$ & $\begin{array}{l}\text { financière des } \\
\text { arties prenantes } \\
\text { tations, besoin de } \\
\text { ères }\end{array}$ \\
\hline
\end{tabular}

Exigences de durabilité : nécessité d’un lien entre des activités habituellement cloisonnées pour créer des filières circulaires, volonté de valorisation des déchets et coproduits

Impacts en termes de DD : fort impact sur le dynamisme territorial, impact économique, environnemental et social faible au sein des frontières mais participe au développement de projets de bouclage de flux

\section{Tableau 2. Caractéristiques communes aux BMC non marchands}

La comparaison de ces deux modèles, marchands et non marchands, permet de souligner la complémentarité des initiatives économiques qui mettent en œuvre l'EC. Les éléments communs de ces modèles appuient les propos de Micheaux et Aggeri [MIC 17] : les BMC ont pour objectif de boucler les flux de matière et d'énergie; pour y parvenir ils sont nécessairement connectés à des réseaux d'acteurs diversifiés. Les BMC dépendent donc de leur environnement. Cette recherche met en lumière l'idée selon laquelle chaque acteur 
économique est spécialisé dans son secteur d'activité, alors que la valorisation des sousproduits et des déchets est souvent trans-sectorielle : les déchets trouvent un débouché dans un autre secteur, inconnu de l'entreprise productrice. Dans l'économie linéaire, ces secteurs sont cloisonnés. Ainsi, des structures d'appui et d'accompagnement sont nécessaires pour mettre en réseau ces acteurs et leur permettre d'exploiter au mieux leur potentiel synergique. Cette recherche permet donc d'appréhender la complémentarité des acteurs, et de percevoir chaque structure économique comme un maillon au sein d'une ou plusieurs filières circulaires.

Notre étude de cas permet de montrer l'aspect multidimensionnel et composite de la valeur créée par ces initiatives. Chacun à leur manière, ces BMC génèrent une valeur économique, sociale, environnementale et territoriale. Cependant, les deux cas étudiés dont les BM sont non marchands rencontrent une certaine fragilité financière, et appellent un soutien public pour réaliser leurs missions de coordination.

Par ailleurs, il est à noter que les initiatives d'EC s'inscrivent dans un contexte local mais que les frontières géographiques des projets sont amenées à évoluer, notamment à cause de la localisation et de la disponibilité des gisements de coproduits.

\section{Discussion et conclusions}

Nous nous sommes intéressés dans cette recherche aux modèles économiques permettant de créer de la valeur dans des projets d'économie circulaire. Sur la base de la littérature et des études de cas menées, nous avons mis en évidence les nouveaux mécanismes de création de valeur à partir des business models circulaires et des composants du processus de création de valeur dans le secteur agricole.

Outre la distinction entre modèle marchand et non marchand, cette étude permet de faire le lien entre les spécificités des produits agricoles et les impacts sur les BMC dans ce secteur, et ainsi de comprendre quelles pratiques managériales sont pertinentes pour assurer le développement de ce type de BM. Les acteurs rencontrés s'accordent sur la nécessité absolue d'anticiper le risque de contamination (pathogènes, substances toxiques) via des prétraitements adaptés aux coproduits à valoriser. La saisonnalité des coproduits agricoles nécessitent une grande flexibilité des ressources humaines et des outils de production. Dans le cas de Grap'Sud, les heures des salariés sont annualisées afin de permettre une large mobilisation lors de la période post-vendanges, les phases de maintenance des infrastructures sont organisées lors des périodes de faible activité. La périssabilité des produits, et surtout la dégradation rapide des molécules d'intérêt impliquent de traiter la matière fraîche rapidement. Il s'agit là d'un défi majeur pour les BMC de ce secteur. En effet, les périodes de production sont courtes et saisonnières alors que la demande du marché, construite par des produits de synthèse, est régulière. Les secteurs de l'énergie, la pharmaceutique, la neutraceutique et de la cosmétique sont demandeurs de nouveaux produits biosourcés mais pour parvenir à nouer une relation à long-terme, les BMC doivent s'adapter aux contraintes de ces secteurs et être en mesure de fournir des produits toute l'année. Pour cela, une taille critique et une capacité de stockage pour des produits intermédiaires stabilisés sont indispensables. Des volumes suffisants de coproduits sont nécessaires pour atteindre cette taille critique, mais plus l'unité de valorisation tend à élargir géographiquement son périmètre de collecte, plus les coûts de la logistique inversée 
augmentent et plus l'impact environnemental est élevé. L'objectif est de trouver l'échelle géographique optimale pour atteindre la taille critique permettant des coûts logistiques bas et des impacts environnementaux limités. De plus, en raison de la périssabilité et de la nécessité de fournir des produits toute l'année, la gestion du stock est fondamentale dans ces BMC. Par ailleurs, la typicité des ressources agricoles doit être prise en compte lorsque qu'une initiative souhaite s'étendre géographiquement : les caractéristiques des coproduits et les molécules d'intérêt peuvent varier. L'entreprise doit donc adapter localement ses procédés. Enfin, les initiatives étudiées ont montré que la variabilité des prix peut être compensée par une approche multi-ressources : Grap'Sud étend ses procédés à plusieurs coproduits de fruits et Bâtir en Balles propose de nouvelles voies de valorisation pour différentes variétés de céréales. La diversification de l'entreprise vers la valorisation de différents coproduits agricoles permet de diminuer le risque financier et de lisser les prix de ventes. En choisissant des gisements à valoriser ayant des saisonnalités différentes, la diversification permet également d'optimiser l'usage des ressources techniques et d'éviter les périodes de faible production.

Nous avons souligné l'importance de la diversité et de la complémentarité des initiatives pour créer des systèmes circulaires. Nous avons constaté le caractère trans-sectoriel de la valorisation des agro-déchets comme, par exemple, les liens entre le secteur du vin et celui des cosmétiques. Ceci nous amène à nous interroger sur la pertinence de l'approche filière classique constituée d'une succession d'opérations subies par une matière première. En effet, il apparaît primordial de créer des liens entre différentes filières pour développer des procédés et des applications innovantes, et ainsi de permettre la transition d'une économie linéaire vers une économie circulaire.

Le rôle des structures de support n'est que très peu étudié dans la littérature sur l'EC. Ce manque est d'autant plus préjudiciable à la compréhension de l'EC qu'une partie des limites à la mise en œuvre de stratégies de circularité ne relève pas de contraintes techniques, mais plutôt de problèmes organisationnels ou d'acquisition de capacités et de management des relations humaines [GAL 16]. Bien qu'une étude de plus grande ampleur sur les BMC non marchands soit nécessaire pour valider ce constat, nos résultats montrent que les structures non marchandes de coordination que nous avons étudiées rencontrent des difficultés de financement. Ces structures de support ont pour objectif d'assurer la coordination entre différents acteurs, pour mettre en œuvre des stratégies d'EC, ou de soutenir le développement de procédés de valorisation nouveaux; elles sont donc complémentaires et nécessaires au bon développement des structures marchandes mais ne parviennent pas à faire reconnaître leur utilité et à obtenir les financements nécessaires pour couvrir leurs coûts de fonctionnement. Des subventions peuvent être accordées au lancement, mais seulement pour une période temporaire. Il est ensuite attendu que la structure parvienne à générer de la valeur économique pour assurer sa pérennité. Ainsi le développement d'une initiative d'EC semble conditionné par un intérêt financier ; la seule volonté de générer de la valeur environnementale ou sociale ne suffit pas à pérenniser ces initiatives sur le long terme. Il apparaît primordial d'identifier pour chaque initiative la valeur apportée aux populations cibles ainsi que la valeur partagée qui en résulte, tant en termes social, qu'environnemental et territorial; et de réussir à souligner l'importance de cette valeur pour justifier l'intérêt de financer l'initiative. Il est à noter que trois des cas étudiés ont été mis en place à la suite d'initiatives privées tandis que le cas Bio-Vallée Lauragais a été initié par 
des collectivités territoriales. On remarque ainsi que le développement de l'économie circulaire sur un territoire est source d'opportunités entrepreneuriales (individuelle ou collective) qui émanent d'acteurs privés et/ou publics, créant ainsi une dynamique territoriale synonyme de développement local.

En effet, les initiatives d'EC se développent pour répondre à des enjeux locaux et l'un des objectifs de l'EC est de mettre en place des bouclages de flux les plus courts possibles pour limiter l'impact environnemental. Cependant, certaines infrastructures de traitement des déchets doivent faire face à des contraintes liées aux économies d'échelle. Ainsi le dimensionnement des unités de traitement est conçu pour traiter d'importants volumes de matières. Cela oblige les acteurs à s'approvisionner parfois très loin ce qui remet en cause l'esprit même de l'EC. Une réflexion sur la taille des unités de traitement devrait être menée dans de futures recherches.

Enfin, les différents cas étudiés permettent de souligner l'importance de l'innovation technologique dans le cadre de la valorisation de coproduits. En effet, transformer des déchets en ressources nécessite des procédés spécifiques, ce qui implique des investissements en R\&D et le développement d'infrastructures adaptées.

\section{Bibliographie}

[ANT 16] ANTIKAinen M., VALKOKARi K., «A framework for sustainable circular business model innovation », Technology Innovation Management Review, $\mathrm{n}^{\circ}$ 6, p. 5-12, 2016.

[ARN 16] ARNSPERGER C., Bourg D., «Vers une économie authentiquement circulaire », Revue de l'OFCE, No. 145, p. 91-125, 2016.

[BAR 05] BARLES S., L'invention des déchets urbains - France: 1790-1970, Editions Champ Vallon, Seyselles, 2005.

[Ben 16] Ben Chedly H., Brunschwig G., Veysset P., Pierret P., Astier M., Goron J., Chapuis D., «Le concept d'économie circulaire au service des systèmes agricoles comme levier d'optimisation du fonctionnement d'un groupe d'exploitations au niveau d'un territoire », Le Développement territorial sous tous ses Aspect(s)!, Toulouse - Aspet, France, 15 Novembre 2016.

[BEU 18] Beulque R., Aggeri F., Abraham F., Morel S., «Business models circulaires : vers une création et captation de valeur pérenne ? Les enseignements du recyclage et de la réutilisation automobiles », Finance Contrôle Stratégie, NS-1, 1-33, 2018.

[BLO 16] BlOCKPOEL M., Business Performance in the Dutch Circular Economy, 2016.

[CAL 18] CALLOIS J-M., «Economie circulaire dans le monde rural - résultats du Projet CAPDOR» Biomasse et économie circulaire: quels enjeux, quelles opportunités et quels risques?, Salon International de l'Agriculture, Paris, 1 Mars 2018.

[CHA 18] Chambre D’Agriculture D’Occitanie et Cerfrance, Agri'scopie ${ }^{\circledR}$ Occitanie édition 2018, S.l., en ligne : https://occitanie.chambre-agriculture.fr/publications/toutes-les-publications/la-publicationen-detail/actualites/agriscopier-occitanie-edition-2018.

[DEM 10] DeMIL B., LECOCQ X., « Business model evolution: in search of dynamic consistency », Long Range Planning, $\mathrm{n}^{\mathrm{O}} 4$, p. 227-246, 2010.

[ELK 98] ElKingtON J., "Cannibals with forks: the triple bottom line », Measuring Business Excellence, Vol. 2, Issue: 3, p.18-22, 1998. 
[ELL 12] ELLEN MACARTHUR FOUNDATION, Towards the circular economy: economic and business rationale for an accelerated transition, 2013. en ligne http://circularfoundation.org/sites/default/files/tce report1 2012.pdf.

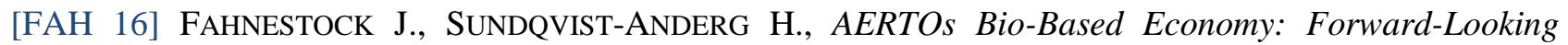
Analysis, S.1., 2016.

[GAL 16] Gallaud D., LAPERChE B., Economie circulaire et développement durable : Ecologie industrielle et circuits courts, ISTE Editions. Collection innovation, entrepreneuriat et gestion, Londres, 2016.

[GAR 17] Garcia Coleta K., Garcia Lopes H., SAlum F., «Business Models: Analysis of its Conceptual and Theoretical Development », Encuentro da ANPAD, São Paulo, 1 Octobre 2017.

[GHI 16] Ghisellini P., Cialani C., Ulgiati S., « A review on circular economy: the expected transition to a balanced interplay of environmental and economic systems », Journal of Cleaner Production, $\mathrm{n}^{\mathrm{o}} 114$, p. 11-32, 2016.

[JON 13] JONCOUX S., «Les "produits résiduaires organiques" pour une intensification écologique de l'agriculture : ressources, déchets ou produits ? : sociologie des formats de valorisation agricole », Thèse de doctorat, Université Toulouse le Mirail - Toulouse II, 2013.

[JOY 16] JOYCE A., PAQUIN R.L., « The triple layered business model canvas: A tool to design more sustainable business models », Journal of Cleaner Production, $\mathrm{n}^{\circ}$ 135, p. 1474-1486, 2016.

[JuR 16] Jurgilevich A., Birge T., Kentala-Lehtonen J., Korhonen-Kurki K., Pietikainen J., SAIKKU L. et SCHÖSLER H., "Transition towards circular economy in the food system », Sustainability, $\mathrm{n}^{\circ} 8$, p. 1-14, 2016.

[LEW 16] LEWANDOWSKI M., « Designing the business models for circular economy-Towards the conceptual framework », Sustainability, n ${ }^{\circ} 8$, p. 43, 2016.

[LIN 17] LINDER M., WILLIANDER M., « Circular business model innovation: inherent uncertainties », Business Strategy and the Environment, $\mathrm{n}^{\mathrm{o}}$ 26, p. 182-196, 2017.

[MEN 14] MENTINK B., Circular business model innovation: a process framework and a tool for business model innovation in a circular economy, Master of Science in industrial ecology, Delft University of technology \& Leiden University, 2014.

[OST 10] OSTERWALDER A., PIGNEUR Y., Business model generation: a handbook for visionaries, game changers, and challengers, John Wiley \& Sons, Hoboken, New Jersey, 2010.

[RAS 07] RASMUSSEN B., Business Models and the Theory of the Firm, Pharmaceutical Industry Project Working Paper, Centre for Strategic Economic Studies, Victoria University, Melbourne, $\mathrm{n}^{\mathrm{o}} 32$, p. 1-11, 2007.

[RID 12] RIDDER H-G, « Book review - Case study research: design and methods. », German Journal of Human Resource Management, $\mathrm{n}^{\circ}$ 26, p. 93-95, 2012.

[RIE 09] RIEUTORT L., Dynamiques rurales françaises et re-territorialisation de l'agriculture, L'Information géographique, Vol. 73, nº. 1, p. 30-48, 2009.

[ROO 17] Rood T., Muilwijk H., Westhoek H., Food for the circular economy, PBL Netherlands Environmental Assessment Agency, No. 2878, The Hague, Pays-bas, 2017.

[SCH 13] SCHUlte UWE G., «New business models for a radical change in resource efficiency », Environmental innovation and societal transitions, vol. 9, p. 43-47, 2013.

[SEM 14] SEMPELS C., «Implementing a circular and performance economy through business model innovation », dans E.M. Foundation (dir.), A New Dynamic. Effective Business in a Circular Economy, 2014.

[Ulv 14] Ulvenblad P., Hoveskog M., Tell J., Stahl J., Barth H., «Agricultural business model innovation in Swedish food production: the influence of self-leadership and lean innovation », Druid 
Society Conference on Entrepreneurship-Organization-Innovation, Copenhagen Business School, Copenhagen, Danemark, 2014.

[WIR 11] WIRTZ B.W., Business Model Management: Design-Instruments—Success Factors, Gabler, Wiesbaden, 2011.

[YIN 03] YIN R.K., Case study research: design and methods, Sage Publications, Thousand Oaks, 2003.

[YIN 12] YING J., LI-JUN Z., « Study on green supply chain management based on circular economy », Physics Procedia, n $^{\circ}$ 25, p. 1682-1688, 2012. 